\title{
Symmetry in Nonlinear Mechanics: Averaging and Normalization Procedures, New Problems and Algorithms
}

\author{
Alexey K. LOPATIN \\ Institute of Mathematics, National Academy of Science of Ukraine, \\ 3 Tereshchenkivs'ka Street, 252004 Kyïv, Ukraïna, \\ e-mail: mathkiev@sovam.com; fax:38-044-225-2010
}

The idea of introducing coordinate transformations to simplify the analytic expression of a general problem is a powerful one. Symmetry and differential equations have been close partners since the time of the founding masters, namely, Sophus Lie (1842-1899), and his disciples. To this days, symmetry has continued to play a strong role. The ideas of symmetry penetrated deep into various branches of science: mathematical physics, mechanics and so on.

The role of symmetry in perturbation problems of nonlinear mechanics, which was already used by many investigators since the 70-th (G. Hori, A. Camel, U. Kirchgraber), has been developed considerably in recent time to gain further understanding and development such constructive and powerful methods as averaging and normal form methods.

Normalization techniques in the context with the averaging method was considered in works by A.M. Molchanov [1], A.D. Brjuno [2], S.N. Chow, J. Mallet-Paret [3], Yu.A. Mitropolsky, A.M. Samoilenko [4], J.A. Sanders, F. Verhulst [5].

An approach, where Lie series in parameter were used as transformation, was considered in works by G. Hori [6], [7], A. Kamel [8], U. Kirchgraber [10], U. Kirchgraber, E. Stiefel [9], V.N. Bogaevsky, A.Ya. Povzner [11], V.F. Zhuravlev, D.N. Klimov [12].

Asymptotic methods of nonlinear mechanics developed by N.M. Krylov, N.N. Bogolyubov and Yu.A. Mitropolsky and known as the KBM method (see, for example, Bogolyubov N.N. and Mitropolsky Yu.A [18]) is a powerful tool for investigation of nonlinear vibrations.

The present lecture deals with the development of new normalization procedures and averaging algorithms in problems of nonlinear vibrations. Namely, the development of asymptotic methods of perturbation theory is considered, making wide use of group theoretical techniques. Various assumptions about specific group properties are investigated, and are shown to lead to modifications of existing methods, such as the Bogolyubov averaging method and the Poincaré-Birkhoff normal form, as well as to formulation of new ones. The development of normalization techniques on Lie groups is also treated.

Copyright (C) 1996 by Mathematical Ukraina Publisher. All rights of reproduction in any form reserved.

\footnotetext{
${ }^{\star}$ The research presented in this publication was made possible in part by Grant No UB2000 from the
} International Science Foundation. 


\section{Mathematical Background}

Below we are giving a short survey of two methods: Bogolyubov averaging method and normal form method.

1.1. The standard system and Bogolyubov's averaging. The new normalization techniques was developed by Yu.A. Mitropolsky, A.K. Lopatin [13]-[15], A.K. Lopatin $[16],[17]$. In their works, a new method was proposed for investigating systems of differential equations with small parameters. It was a further development of Bogolyubov's averaging method referred to by the authors as "the asymptotic decomposition method". The idea of a new approach originates from Bogolyubov's averaging method (see [18]) but its realization is needed to use essentially new apparatus - the theory of continuous transformation groups.

Let us explain the idea of the new approach. As is known, the starting point of investigation by the averaging method is a system in the standard form

$$
\frac{d x}{d t}=\varepsilon X(x, t, \varepsilon),
$$

where $x=\mathrm{col}\left\|x_{1}, \ldots, x_{n}\right\|, X(x, t, \varepsilon)$ is an $n$-dimensional vector.

System (1), upon averaging

$$
X_{0}(\xi, \varepsilon)=\lim _{T \rightarrow \infty} \frac{1}{T} \int_{0}^{T} X(\xi, t, \varepsilon) d t
$$

and with a special change of variables, is reduced to the averaged system

$$
\frac{d \bar{x}}{d t}=\varepsilon X_{0}^{(1)}(\bar{x})+\varepsilon^{2} X_{0}^{(2)}(\bar{x})+\cdots,
$$

which does not explicitly contain the argument $t$. (To ensure the existence of the average, we impose special conditions on the functions $X_{j}(x, t, \varepsilon), j=\overline{1, n}$. We omit the explicit form of these conditions). Let us rewrite the initial system (1) in the equivalent form

$$
\frac{d x}{d t}=\varepsilon X(x, y, \varepsilon), \quad \frac{d y}{d t}=1
$$

and the averaged system (2) correspondingly in the form

$$
\frac{d \bar{x}}{d t}=\varepsilon X_{0}(\bar{x}), \quad \frac{d \bar{y}}{d t}=1,
$$

where $X_{0}(\bar{x})=X_{0}^{(1)}(\bar{x})+\varepsilon X_{0}^{(2)}(\bar{x})+\cdots$. Integration of (4) is simpler than that of $(3)$, since the variables are separated: the system for slow variables $\bar{x}$ does not contain a fast variable $\bar{y}$ and is integrated independently.

Everything stated above allows us to interpret the averaging method in the following way: the averaging method transforms (3) with nonseparated variables into (4) with fast and slow variables separated.

The described property of the separation of variables with the help of the averaging method has group-theoretical characteristics: the averaging method transforms (3), which 
is not invariant with respect to the one-parameter transformation group generated by a vector field

$$
\mathbf{W}=\frac{\partial}{\partial y}
$$

associated with the system of zero approximation (3), into the averaged system (4), which is invariant with respect to the one-parameter transformation group generated by a vector field

$$
\mathbf{U}=\frac{\partial}{\partial \bar{y}},
$$

associated with the system of zero approximation (4). This statement can be easily proved.

1.2. The normal form method. Consider a system of differential equations with coefficients that are analytic in the neighborhood of zero

$$
\begin{aligned}
& \dot{y}_{1}=a_{11} y_{1}+\cdots+a_{1 n} y_{n}+\sum f_{m_{1} \ldots m_{n}}^{1} y_{1}^{m_{1}} \ldots y_{n}^{m_{n}}, \\
& \ldots \ldots \ldots \\
& \dot{y}_{n}=a_{n 1} y_{1}+\cdots+a_{n n} y_{n}+\sum f_{m_{1} \ldots m_{n}}^{n} y_{1}^{m_{1}} \ldots y_{n}^{m_{n}} .
\end{aligned}
$$

Nonlinear terms in the right-hand sides of system (5) are started with terms not lower than the second order.

We consider the problem of finding an analytic change of variables

$$
y=f(z), \quad z=\left\|z_{1}, \ldots, z_{n}\right\|,
$$

which turns a maximal number of coefficients at nonlinear terms into zero. The limiting case is the linearization of system (5), i.e., transformation of it into

$$
\dot{z}=\mathcal{A} z, \quad \mathcal{A}=\left\|a_{i j}\right\|, \quad i, j=\overline{1, n}
$$

under the action of the variable change (6). Since the procedure pointed out is ultimately reduced to the solvability of linear nonhomogeneous algebraic equations, it turns out that the reduction

$$
(5) \stackrel{y=f(z)}{\longrightarrow}(7)
$$

is not always possible. In the general case, a system of nonlinear differential equations is obtained

$$
\dot{z}=\mathcal{A} z+F(z) .
$$

This system is called a normal form.

We refer to the corresponding nonlinear term in the above equation as resonance. It is clear that only resonance terms remain in the normal form. In particular, linearization by (8) is possible only when there are no resonance terms (see, for example, [12]).

1.3. Generalization of Bogolyubov's averaging method through the symmetry of the standard system. The asymptotic decomposition method is based 
on the group-theoretical interpretation of the averaging method. Consider the system of ordinary differential equations

$$
\frac{d x}{d t}=\omega(x)+\varepsilon \tilde{\omega}(x)
$$

where

$$
\omega(x)=\operatorname{col}\left\|\omega_{1}(x), \ldots, \omega_{n}(x)\right\| ; \tilde{\omega}(x)=\operatorname{col}\left\|\tilde{\omega}_{1}(x), \ldots, \tilde{\omega}_{n}(x)\right\| .
$$

The differential operator associated with the perturbed system (9) can be represented as

$$
\mathbf{U}_{0}=\mathbf{U}+\varepsilon \tilde{\mathbf{U}}
$$

where

$$
\mathbf{U}=\omega_{1} \frac{\partial}{\partial x_{1}}+\cdots+\omega_{n} \frac{\partial}{\partial x_{n}}, \quad \tilde{\mathbf{U}}=\tilde{\omega}_{1} \frac{\partial}{\partial x_{1}}+\cdots+\tilde{\omega}_{n} \frac{\partial}{\partial x_{n}} .
$$

By using a certain change of variables in the form of a series in $\varepsilon$

$$
x=\varphi(\bar{x}, \varepsilon),
$$

system (9) is transformed into a new system

$$
\frac{d \bar{x}}{d t}=\omega(\bar{x})+\sum_{\nu=1}^{\infty} \varepsilon^{\nu} b^{(\nu)}(\bar{x}),
$$

which is referred to as a centralized system. For this system, $\overline{\mathbf{U}}_{0}=\overline{\mathbf{U}}+\varepsilon \tilde{\overline{\mathbf{U}}}$, where

$$
\begin{aligned}
& \overline{\mathbf{U}}=\omega_{1}(\bar{x}) \frac{\partial}{\partial \bar{x}_{1}}+\cdots+\omega_{n}(\bar{x}) \frac{\partial}{\partial \bar{x}_{n}}, \\
& \tilde{\tilde{\mathbf{U}}}=\sum_{\nu=1}^{\infty} \varepsilon^{\nu} \mathbf{N}_{\nu}, \mathbf{N}_{\nu}=b_{1}^{(\nu)}(\bar{x}) \frac{\partial}{\partial \bar{x}_{1}}+\cdots+b_{n}^{(\nu)}(\bar{x}) \frac{\partial}{\partial \bar{x}_{n}} .
\end{aligned}
$$

We impose a condition on the choice of transformations (10) saying that the centralized system (11) should be invariant with respect to the one-parameter transformation group

$$
\bar{x}=e^{s \overline{\mathbf{U}}\left(\bar{x}_{0}\right)} \bar{x}_{0},
$$

where $\bar{x}_{0}$ is the vector of new variables. Therefore, after the change of variables (13), system (11) turns into

$$
\frac{d \bar{x}_{0}}{d t}=\omega\left(\bar{x}_{0}\right)+\sum_{\nu=1}^{\infty} \varepsilon^{\nu} b^{(\nu)}\left(\bar{x}_{0}\right)
$$

which coincides with the original one up to the notations. This means that we have the identities $\left[\overline{\mathbf{U}}, \mathbf{N}_{\nu}\right] \equiv 0$ for $\overline{\mathbf{U}}, \mathbf{N}_{\nu}, \nu=1,2, \ldots$

Presented below is some material which will be needed for understanding the structure of the present lecture as a whole. The essential point in realizing the above-mentioned 
indicated scheme of the asymptotic decomposition algorithm is that transformations (10) are chosen in the form of a series

$$
x=e^{\varepsilon \mathbf{S}} \bar{x},
$$

where

$$
\begin{aligned}
& \mathbf{S}=\mathbf{S}_{1}+\varepsilon \mathbf{S}_{2}+\cdots \\
& \mathbf{S}_{j}=\gamma_{j 1}(\bar{x}) \frac{\partial}{\partial \bar{x}_{1}}+\cdots+\gamma_{j n}(\bar{x}) \frac{\partial}{\partial \bar{x}_{n}} .
\end{aligned}
$$

Coefficients of $\mathbf{S}_{j}, \gamma_{j 1}(\bar{x}), \ldots, \gamma_{j n}(\bar{x})$, are unknown functions. They should be determined by the recurrent sequence of operator equations

$$
\left[\mathbf{U}, \mathbf{S}_{\nu}\right]=\mathbf{F}_{\nu}
$$

The operator $\mathbf{F}_{\nu}, \nu=1,2, \ldots$ is a known function of $\mathbf{U}$ and $\mathbf{S}_{1}, \ldots, \mathbf{S}_{\nu-1}$, obtained on previous steps.

In the case when $\mathbf{S}$ depends upon $\varepsilon$, Lie series (14) is called a Lie transformation. Thus, the application of a Lie transformation as a change of variables enables us to use the technique of continuous transformation groups.

From the theory of linear operators it is known that the solvability of the nonhomogeneous operator equation (15) depends on properties of solutions of the homogeneous equation

$$
\left[\mathbf{U}, \mathbf{S}_{\nu}\right]=0 \text {. }
$$

Operator (12) $\mathbf{N}_{\nu}$ is a projection of the right-hand side of the equation onto the kernel of operator (16), which is determined from the condition of solvability in the sense of the nonhomogeneous equation

$$
\left[\mathbf{U}, \mathbf{S}_{\nu}\right]=\mathbf{F}_{\nu}-\mathbf{N}_{\nu}, \quad \nu=1,2, \ldots
$$

Depending on the way of solving Eqs. (15)-(17), various modifications of the algorithm of the asymptotic decomposition one are obtained.

The asymptotic decomposition method, being applied to the same objects as the classical asymptotic method, yields identical results. However, the algorithm of the asymptotic decomposition method is, in essence, simpler.

The principal conclusion that can be arrived at after a comparison of the two methods is the following. In the asymptotic decomposition method, the operation of averaging, which is used in Bogolyubov's averaging method, is a certain way of constructing the projection pr $\mathbf{F}$ of the operator $\mathbf{F}$.

In the asymptotic decomposition method, the centralized system is a direct analog of the averaged system of Bogolyubov's averaging method.

We refer to the averaging operation used in the asymptotic decomposition method to construct the projection of an operator onto the algebra of the centralizer as the Bogolyubov projector. 
The last statement means the following. Let us apply the asymptotic decomposition method to Bogolyubov's system in the standard form (3). Let us write out the operator $\mathbf{F}_{\nu}$ of the right-hand side of (15) as

$$
\mathbf{F}_{\nu}=f_{\nu 1}(x, y) \frac{\partial}{\partial x_{1}}+\cdots+f_{\nu n}(x, y) \frac{\partial}{\partial x_{n}} .
$$

Define the Bogolyubov projection of the operator $\operatorname{pr} \mathbf{F}_{\nu}$ as

$$
\operatorname{pr} \mathbf{F}_{\nu}=\left\langle f_{\nu 1}(x, y)\right\rangle \frac{\partial}{\partial x_{1}}+\cdots+\left\langle f_{\nu n}(x, y)\right\rangle \frac{\partial}{\partial x_{n}},
$$

where

$$
\left\langle f_{\nu k}(x, y)\right\rangle={ }_{\operatorname{def}} f_{\nu k}^{0}(x)
$$

is an average value for coefficients $f_{\nu k}$. This notion requires exact definition.

In Bogolyubov's averaging method an average value is understood as

$$
\lim _{T \rightarrow \infty} \frac{1}{T} \int_{0}^{T} f_{\nu k}(x, s) d s=f_{\nu k}^{0}(x)<+\infty, \quad k=1, n .
$$

In our further exposition, definition (18) will be understood as an average value on the group.

We hope that such a preview will make the main part of the lecture easier to follow. We illustrate a further exposition of the material in the next two Subsections using two physically motivated examples: nonlinear oscillators in plane and motion of a point on a sphere. There are classical results for the first example and one can compare them with the present approach. The second example is nontrivial as it cannot be considered by existing methods in a similar way.

\section{Examples: Models Connected with a Nonlinear Oscillator in a Plane}

2.1. Algorithm of asymptotic decomposition method in the space of homogeneous polynomials (group GL(2)). Along with the linear space $V$ over $P$ generated by the elements $x_{1}, \ldots, x_{n}$, we consider the linear space $V_{\otimes \nu}$ over $P$, which is equal to the direct product of the space $V$ taken $\nu$ times.

The vector row composed of the basis elements of $V_{\otimes \nu}$ is denoted by $\hat{\hat{x}}_{m_{\nu}}$. It is evident that $m_{1}=n$ and

$$
\hat{\hat{x}}_{m_{1}}=\left\|x_{1}, \ldots, x_{n}\right\| \text {. }
$$

Let $\mathcal{Q}$ be a constant matrix of dimension $m_{\nu} \times n$ with the elements $q_{i j} \in P$, where $i=\overline{1, m_{\nu}}, j=\overline{1, n}$, and $q_{1}, \ldots, q_{n}$ are the rows as matrix elements in the equality

$$
q=\hat{\hat{x}}_{m_{\nu}} \mathcal{Q}, \quad q=\operatorname{def}\left\|q_{1}, \ldots, q_{n}\right\| .
$$

For an arbitrary sequence of matrices $\mathcal{Q}$, the totality of differential operators

$$
\mathbf{X}=q_{1} \frac{\partial}{\partial x_{1}}+\cdots+q_{n} \frac{\partial}{\partial x_{n}}, \quad q_{i} \in V_{\otimes \nu},
$$


yields the linear space over $P$ which is denoted by $\mathcal{B}\left(V_{\otimes \nu}\right)$. The matrix $\mathcal{Q}$ will be called $a$ matrix of the operator $\mathbf{X}$.

Consider the system of two equations of the first order

$$
\dot{x}_{1}^{\prime}=x_{2}^{\prime} ; \quad \dot{x}_{2}^{\prime}=-x_{1}^{\prime}+\varepsilon\left(1-x_{1}^{\prime 2}\right) x_{2}^{\prime} .
$$

The differential operator associated with system (19) is

$$
\mathbf{U}_{0}^{\prime}=\mathbf{U}^{\prime}+\varepsilon \widetilde{\mathbf{U}}^{\prime},
$$

where

$$
\mathbf{U}^{\prime}=x_{2}^{\prime} \frac{\partial}{\partial x_{1}^{\prime}}-x_{1}^{\prime} \frac{\partial}{\partial x_{2}^{\prime}} ; \quad \tilde{\mathbf{U}}^{\prime}=\left(x_{2}^{\prime}-x_{1}^{\prime 2} x_{2}^{\prime}\right) \frac{\partial}{\partial x_{2}^{\prime}} .
$$

Write these operators in the form

$$
\mathbf{U}^{\prime}=\hat{\hat{x}}_{m_{1}}^{\prime} \mathcal{F} \partial^{\prime}, \mathcal{F}=\left\|\begin{array}{cc}
0 & -1 \\
1 & 0
\end{array}\right\|
$$

Represent the operator $\tilde{\mathbf{U}}$ as the sum

$$
\tilde{\mathbf{U}}^{\prime}=\tilde{\mathbf{U}}_{\otimes 1}^{\prime}+\tilde{\mathbf{U}}_{\otimes 3}^{\prime} \quad \tilde{\mathbf{U}}_{\otimes i}^{\prime} \in \mathcal{B}\left(V_{\otimes i}\right), i=1,3,
$$

where

$$
\tilde{\mathbf{U}}_{\otimes 1}^{\prime}=\hat{\hat{x}}_{m_{1}}^{\prime} \mathcal{Q}_{m_{1}, 1} \partial, \quad \tilde{\mathbf{U}}_{\otimes 3}^{\prime}=\hat{\hat{x}}_{m_{3}}^{\prime} \mathcal{Q}_{m_{3}, 1} \partial
$$

Calculate two approximations in the transformed operator (12)

$$
\mathbf{U}_{0}^{\prime}=\mathbf{U}^{\prime}+\varepsilon \mathbf{N}_{1}^{\prime}+\varepsilon^{2} \mathbf{N}_{2}^{\prime}
$$

Calculate the operators $\mathbf{S}_{1}$ and $\mathbf{S}_{2}$, which can be obtained from the equations

$$
\begin{aligned}
& {\left[\mathbf{U}, \mathbf{S}_{1}\right]=\tilde{\mathbf{U}}-\operatorname{pr} \tilde{\mathbf{U}}} \\
& {\left[\mathbf{U}_{1}, \mathbf{S}_{2}\right]=\left\{-\left[\tilde{\mathbf{U}}, \mathbf{S}_{1}\right]-\frac{1}{2}\left[\mathbf{S}_{1},\left[\mathbf{U}, \mathbf{S}_{1}\right]\right]\right\}-\operatorname{pr}\{\cdots\}}
\end{aligned}
$$

upon the change of variables (14). Solve these equations in two steps. First, we find $\mathbf{S}_{1}$ :

$$
\mathbf{S}_{1} \equiv \mathbf{S}_{\otimes 11}+\mathbf{S}_{\otimes 31}, \mathbf{S}_{\otimes i 1} \in \mathcal{B}\left(V_{\otimes i}\right), i=1,3,
$$

where $\mathbf{S}_{\otimes i 1} \equiv \hat{\hat{x}}_{m_{i}} \Gamma_{1 i} \partial, i=1,3 ; \Gamma_{1 i}$ are rectangular matrices of the dimensions $m_{i} \times n$, which are solutions of the system of independent algebraic equations

$$
\mathcal{F}_{i} \Gamma_{1 i}-\Gamma_{1 i} \mathcal{F}=\mathcal{Q}_{m_{i}, 1}-\operatorname{pr} \mathcal{Q}_{m_{i}, 1}, \mathcal{F}=\mathcal{A}^{T}, i=1,3 .
$$

At the second step, we find $\mathbf{S}_{2}$. We can see that $\mathbf{S}_{2} \in \mathcal{B}\left(V_{\otimes 5}\right)$ implies the structure of the right-hand parts of Eq. (20). We have to find a solution in the form of the sum

$$
\mathbf{S}_{2}=\sum_{i=1}^{5} \mathbf{S}_{\otimes i 2}, \mathbf{S}_{\otimes i 2}=\hat{\hat{x}}_{m_{i}} \Gamma_{2 i} \partial, i=\overline{1,5},
$$


where $\Gamma_{2 i}$ are solutions of the system of algebraic equations

$$
\mathcal{F}_{i} \Gamma_{2 i}-\Gamma_{2 i} \mathcal{F}=\mathcal{Q}_{m_{i}, 2}-\operatorname{pr} \mathcal{Q}_{m_{i}, 2}, i=\overline{1,5} .
$$

Conduct the necessary calculations for the first approximation. Consider Eq. (21) with the matrices $\mathcal{F}_{1}, \mathcal{F}_{2}, \mathcal{F}_{3}$ of the representation of the operator $\mathbf{U}$ in the subspaces $V_{\otimes 1}, V_{\otimes 2}, V_{\otimes 3}$.

Pass from Eqs. (21) to the equations in the spaces $\hat{R}^{\left(m_{1}, n\right)}, \hat{R}^{\left(m_{2}, n\right)}$

$$
G_{\mathcal{F}}^{(i)} \hat{\Gamma}_{i 1}=\hat{\mathcal{Q}}_{m_{i}, 1}-\operatorname{pr} \hat{\mathcal{Q}}_{m_{i}, 1}
$$

where

$$
G_{\mathcal{F}}^{(i)}=\mathcal{F}_{i} \otimes \mathcal{E}_{2}-\mathcal{E}_{m_{i}} \otimes \mathcal{F}^{T}, i=1,3
$$

$\hat{\Gamma}_{1 i}, \hat{\mathcal{Q}}_{m_{i}, 1}$ are vector columns composed of rows of the matrices $\Gamma_{1 i}, \mathcal{Q}_{m_{i}, 1}$.

Taking into account that the difference $\hat{\mathcal{Q}}_{m_{i}, 1}-\hat{\mathcal{Q}}_{m_{i}, 1 N}$ belongs to the image $T_{\mathcal{F}}^{(i)}$ of the operator $G_{\mathcal{F}}^{(i)}$ and is orthogonal to the kernel of the operator $G_{\mathcal{F}}^{(i) T}$, we obtain the system of linear algebraic equations for finding pr $\hat{\mathcal{Q}}_{m_{i}, 1}$.

Finally, we have the operator $\mathbf{U}_{0}$ in the first approximation:

$$
\mathbf{U}_{0}=\mathbf{U}+\varepsilon \mathbf{N}_{1},
$$

where

$$
\begin{aligned}
& \mathbf{N}_{1}=\operatorname{pr} \tilde{\mathbf{U}}=\mathbf{N}_{\otimes 11}+\mathbf{N}_{\otimes 13} ; \\
& \mathbf{N}_{\otimes 11}=\hat{\hat{x}}_{m_{1}} \mathcal{Q}_{m_{1}, 1 N} \partial=\frac{1}{2}\left(x_{1} \frac{\partial}{\partial x_{1}}+x_{2} \frac{\partial}{\partial x_{2}}\right) ; \\
& \mathbf{N}_{\otimes 31}=\hat{\hat{x}}_{m_{3}} \mathcal{Q}_{m_{3}, 1 N} \partial=-\frac{1}{8}\left(\left(x_{1}^{2}+x_{2}^{2}\right) x_{1} \frac{\partial}{\partial x_{1}}+\left(x_{1}^{2}+x_{2}^{2}\right) x_{2} \frac{\partial}{\partial x_{2}}\right) .
\end{aligned}
$$

After similar calculations, we find the centralized system in the second approximation

$$
\begin{aligned}
\frac{d x_{1}}{d t}= & x_{2}+\varepsilon\left(\frac{1}{2}-\frac{1}{8}\left(x_{1}^{2}+x_{2}^{2}\right)\right) x_{1}+ \\
& \varepsilon^{2}\left(-\frac{1}{4}+\frac{3}{8}\left(x_{1}^{2}+x_{2}^{2}\right)-\frac{11}{128}\left(x_{1}^{2}+x_{2}^{2}\right)^{2}\right) x_{2} \\
\frac{d x_{2}}{d t}= & -x_{1}+\varepsilon\left(\frac{1}{2}-\frac{1}{8}\left(x_{1}^{2}+x_{2}^{2}\right)\right) x_{2}- \\
& \varepsilon^{2}\left(-\frac{1}{4}+\frac{3}{8}\left(x_{1}^{2}+x_{2}^{2}\right)-\frac{11}{128}\left(x_{1}^{2}+x_{2}^{2}\right)^{2}\right) x_{1} .
\end{aligned}
$$

We can easily see that upon transformation of the variables by formulae

$$
y_{1}=\sqrt{x_{1}^{2}+x_{2}^{2}}, y_{2}=\arctan \frac{x_{1}}{x_{2}} .
$$


the centralized system takes the form

$$
\begin{aligned}
& \frac{d y_{1}}{d t}=\varepsilon\left(\frac{1}{2}-\frac{1}{8} y_{1}^{2}\right) y_{1} \\
& \frac{d y_{2}}{d t}=1-\varepsilon^{2}\left(\frac{1}{4}-\frac{3}{8} y_{1}^{2}+\frac{11}{128} y_{1}^{4}\right) .
\end{aligned}
$$

To pass to the solution of the initial Eqs. (19) in the second approximation, we have to know the operator $\mathbf{S}_{2}$. Calculation of $\mathbf{S}_{2}$ is analogous to that of $\mathbf{S}_{1}$.

2.2. Procedures of normalization in the representation spaces of the groups $\mathrm{GL}(2)$ and $\mathrm{SO}(2)$. Consider the nonlinear oscillator (1). All considerations of Subsection 2.1 were based upon the invariance property of the subspaces $V_{\otimes 1}, V_{\otimes 2}, \ldots$ which is associated with the system of zero approximation. The fact of invariance is expressed by the relation

$$
\mathbf{U} \hat{\hat{x}}_{m_{j}}=\hat{\hat{x}}_{m_{j}} \mathcal{F}_{j}, \quad j=1,2, \ldots
$$

where $\mathcal{F}_{j}$ is the representation matrix of $\mathbf{U}$ in the subspace $V_{\otimes j}$.

A natural question arises: are subspaces $V_{\otimes 1}, V_{\otimes 2}, \ldots$ unique invariant subspaces in the linear space of homogeneous polynomials? It turns out that they are not.

Consider the linear space $T_{\otimes}$ that is the direct sum of subspaces $T_{\otimes 1}, T_{\otimes 2}, T_{\otimes 3}, \ldots$ with the bases

$$
\begin{aligned}
& f^{\left(m_{1}\right)}=\left\|x_{1}, x_{2}\right\|, \\
& f^{\left(m_{2}\right)}=\left\|2 x_{1} x_{2}, x_{2}^{2}-x_{1}^{2}\right\|, \\
& f^{\left(m_{3}\right)}=\left\|3\left(x_{1}^{2}+x_{2}^{2}\right) x_{1}-4 x_{1}^{3}, 4 x_{2}^{3}-3\left(x_{1}^{2}+x_{2}^{2}\right) x_{2}\right\|,
\end{aligned}
$$

It is easy to verify that each subspace $T_{\otimes j}$ is turned into itself by $\mathbf{U}$, i.e., is invariant with respect to it. To do so, it is sufficient to find representation matrices of $\mathbf{U}$ in these subspaces

$$
\mathbf{U} f^{\left(m_{j}\right)}=f^{\left(m_{j}\right)} \mathcal{F}_{j}, \quad \mathcal{F}_{j}=\left\|\begin{array}{cc}
0 & -j \\
j & 0
\end{array}\right\| .
$$

For a better understanding of the structure of the space $T_{\otimes}$, let us introduce new variables $\rho$ and $\varphi$ by the formula

$$
x_{1}=\rho \sin \varphi, \quad x_{2}=\rho \cos \varphi .
$$

In new variables, basis vectors (22) are written down as follows

$$
\hat{\hat{\varphi}}_{m_{k}}=\left\|\rho^{k} \sin k \varphi, \rho^{k} \cos k \varphi\right\|, \quad k=1,2, \ldots
$$

So, passing to the space $T_{\otimes} \subset T(V)$ means passing from the space of homogeneous polynomials in two variables to the space of trigonometric functions (Fourier series). 
The just-described process of choosing a new representation space for the operator $\mathbf{U}$ has a deep group-theoretical background. Let us consider it in detail.

Consider the set of four linearly independent operators

$$
\mathbf{V}_{11}=x_{1} \frac{\partial}{\partial x_{1}}, \mathbf{V}_{12}=x_{1} \frac{\partial}{\partial x_{2}}, \mathbf{V}_{21}=x_{2} \frac{\partial}{\partial x_{1}}, \mathbf{V}_{22}=x_{2} \frac{\partial}{\partial x_{2}}
$$

which generate a complete linear finite-dimensional Lie algebra $\mathcal{B}_{G L(2)}$ of order four. From (23), a general linear group $G L(2)$ is restored. To write out the elements of this group in explicit form, let us write down its general element through a Lie series

$$
x^{\prime}=\exp \mathbf{V} x,
$$

where

$$
\mathbf{V}=s_{11} \mathbf{V}_{11}+s_{12} \mathbf{V}_{12}+s_{21} \mathbf{V}_{21}+s_{22} \mathbf{V}_{22}
$$

$s_{11}, s_{12}, s_{21}, s_{22}$ are group parameters which range in the neighborhood of zero.

We write down the series (24) in the finite form as

$$
x^{\prime}=x e^{\mathcal{F}_{1}(s)} .
$$

The matrix $\mathcal{G}(s)=e^{\mathcal{F}_{1}(s)}$, where the matrix $\mathcal{F}_{1}$ is a representation matrix of $\mathbf{V}$ in the subspace $V_{\otimes 1}$, determines the general element of $G L(2)$.

In light of the above considerations, we may say that the linear space of homogeneous polynomials $T(V)$ is a representation space for the general linear group $G L(n), n=2$.

The operator $\mathbf{U}$ of the system of zero approximation generates the rotation group $S O(2)$ in the plane. To find the explicit form of the elements of this group, we also make use of a Lie series

$$
x^{\prime}=\exp (\varphi \mathbf{U}) x .
$$

After the corresponding computations, we come to the result

$$
\left\|\begin{array}{l}
x_{1}^{\prime} \\
x_{2}^{\prime}
\end{array}\right\|=\left\|\begin{array}{cc}
\cos \varphi & \sin \varphi \\
-\sin \varphi & \cos \varphi
\end{array}\right\|\left\|x_{1}\right\| .
$$

Thus, the linear space of the trigonometric functions $T_{\otimes}$ is the representation space for the rotation group $S O(2)$ in the plane. Let us denote this space by $T_{S O(2)}$.

In the normal form method, the representation space for a general linear group $G L(n)$ is chosen as a representation space. In the asymptotic decomposition method, the representation space for the subgroup of the same $G L(n)$, is chosen as a representation space.

So, the normal form method, which makes use of a universal representation space of the general linear group, does not consider the true algebraic structure of the system of zero approximation.

Contrary to that, the asymptotic decomposition method is based essentially on a deep connection between the representation theory for continuous groups and special functions of mathematical physics. This theory has been intensively developed during the last decades (see Vilenkin N.Ya. [19], Barut A., Roczka R. [20]).

2.3. Asymptotical decomposition algorithm for a perturbed motion on $\mathbf{S O}(2)$. Let us consider the Van der Pol (19) system as a perturbed motion on $S O(2)$. The 
system of zero approximation (19) yields the group $S O(2)$. Pass to the polar coordinates in (19)

$$
x_{1}=\rho \sin \varphi, x_{2}=\rho \cos \varphi .
$$

Finally, we obtain $\mathbf{U} \equiv \partial / \partial \varphi$.

The operator $\mathbf{U}$ has the representation matrix $\mathcal{F}_{m_{n}}$ in the subspace $T_{\otimes n}$. This matrix can be calculated by

$$
\mathbf{U} f^{(n)}=f^{(n)} \mathcal{F}_{m_{n}}, \mathcal{F}_{m_{n}}=\left\|\begin{array}{rr}
0 & -n \\
n & 0
\end{array}\right\| .
$$

Illustrate application of the asymptotic decomposition method to system (1) in the representation space of $T_{\otimes}$. Calculate only the first approximation. Let one term $\mathbf{S}_{1}$ be in the transformation (14) and the transformed operator be represented by the sum

$$
\mathbf{U}_{0}=\mathbf{U}+\varepsilon \mathbf{N}_{1} \text {. }
$$

According to the general theory, we should consider the equation

$$
\left[\mathbf{U}, \mathbf{S}_{1}\right]=\mathbf{F}_{1}, \quad \mathbf{F}_{1}={ }_{\text {def }} \tilde{\mathbf{U}} .
$$

After a change of variables (25), $\partial / \partial x_{1}, \partial / \partial x_{2}$ turn into $\mathbf{L}_{1}, \mathbf{L}_{2}$, respectively

$$
\mathbf{L}_{1}=\sin \varphi \frac{\partial}{\partial \rho}+\frac{\cos \varphi}{\rho} \frac{\partial}{\partial \varphi}, \quad \mathbf{L}_{2}=\cos \varphi \frac{\partial}{\partial \rho}-\frac{\sin \varphi}{\rho} \frac{\partial}{\partial \varphi} .
$$

Rewrite $\mathbf{U}, \widetilde{\mathbf{U}}$ in new variables using $\mathbf{L}_{1}, \mathbf{L}_{2}$

$$
\begin{aligned}
& \mathbf{U} \equiv \frac{\partial}{\partial \varphi} \equiv\|\rho \sin \varphi, \rho \cos \varphi\| \mathcal{F} L, \\
& \widetilde{\mathbf{U}}=\|\rho \sin \varphi, \rho \cos \varphi\| \mathcal{Q}_{11} L+\left\|\rho^{3} \sin 3 \varphi, \rho^{3} \cos 3 \varphi\right\| \mathcal{Q}_{31} L .
\end{aligned}
$$

Write the operator of the transformation $\mathbf{S}_{1}$ following the structure of the right-hand part of Eq. (26) in the form

$$
\mathbf{S}_{1}=\mathbf{S}_{11}+\mathbf{S}_{31}
$$

where

$$
\mathbf{S}_{11}=\|\rho \sin \varphi, \rho \cos \varphi\| \Gamma_{11} L, \mathbf{S}_{31}=\left\|\rho^{3} \sin 3 \varphi, \rho^{3} \cos 3 \varphi\right\| \Gamma_{31} L,
$$

$\Gamma_{11}, \Gamma_{31}$ are a unknown second-order square matrices. In the general case, they depend on the variable $\rho$.

Substituting $\mathbf{U}, \widetilde{\mathbf{U}}$ and $\mathbf{S}_{1}$ into Eq. (26), we obtain two independent subsystems of linear algebraic equations

$$
\mathcal{F}_{1} \Gamma_{j 1}-\Gamma_{j 1} \mathcal{F}=\mathcal{Q}_{j 1}, j=1,2 .
$$

All the further calculations are similar to ones done in the previous subsection. We will give the final results. 
Thus, the operator $\mathbf{N}_{1}$ defined by the matrix can be written in the final form

$$
\mathbf{N}_{1} \equiv\left(\frac{1}{2}-\frac{1}{8} \rho^{2}\right) \rho \sin \varphi \mathbf{L}_{1}+\left(\frac{1}{2}-\frac{1}{8} \rho^{2}\right) \rho \cos \varphi \mathbf{L}_{2} \equiv \rho\left(\frac{1}{2}-\frac{1}{8} \rho^{2}\right) \frac{\partial}{\partial \rho} .
$$

By the operator $\mathbf{U}_{0}=\mathbf{U}+\varepsilon \mathbf{N}_{1}$, we restore the centralized system of the first approximation

$$
\dot{\rho}=\frac{\varepsilon}{2}\left(1-\frac{1}{4} \rho^{2}\right) \rho, \dot{\varphi}=1 .
$$

Comparison of the asymptotic decomposition algorithm in the representation space $T_{\otimes}$ of trigonometrical functions described in this Subsection with the analogous algorithm in the space of polynomials $T(V)$ in the previous subsection shows a substantial decrease in calculating effort. This fact takes place due to lowering the order of the representation matrices $\mathcal{F}_{j}$ of the operator $\mathbf{U}$ in the subspaces $T_{\otimes j}$ in comparison with the subspace $V_{\otimes j}$. Really, in the first case, the order of the matrices $\mathcal{F}_{j}$ is unchangeable and is equal to 2 . In the second case, it grows proportionally to the index $j$.

Finally, let us compare the asymptotic decomposition algorithm with existing methods. If the representation space $T_{\otimes}$ of the group $S O(2)$ is chosen, we then obtain the results of the Krylov-Bogolyubov asymptotic method. If the representation space $V_{\otimes}$ of the general linear group $G L(2)$ (the space of homogeneous polynomials) is chosen, then we obtain the results of the normal forms method.

2.4. Group averaging for a perturbed motion on $\mathrm{SO}(2)$. Consider the secondorder system

$$
\dot{y}_{1}=y_{2}, \quad \dot{y}_{2}=-y_{1}-\varepsilon y_{1}^{3},
$$

which is equivalent to the Duffin equation.

After the change of variables

$$
y_{1}=\rho^{\prime} \sin \varphi^{\prime}, \quad y_{2}=\rho^{\prime} \cos \varphi^{\prime},
$$

system (27) is turned into a system of standard form

$$
\begin{aligned}
& \dot{\rho}^{\prime}=-\varepsilon\left(\rho^{\prime}\right)^{3}\left(-\frac{1}{8} \sin 4 \varphi^{\prime}+\frac{1}{4} \sin 2 \varphi^{\prime}\right), \\
& \dot{\varphi}^{\prime}=1+\varepsilon\left(\rho^{\prime}\right)^{2}\left(\frac{3}{8}+\frac{1}{8} \cos 4 \varphi^{\prime}-\frac{1}{2} \cos 2 \varphi^{\prime}\right) .
\end{aligned}
$$

Write down the operator $\mathbf{U}_{0}^{\prime}$ associated with system (28)

$$
\mathbf{U}_{0}^{\prime}=\mathbf{U}_{1}^{\prime}+\varepsilon \tilde{\mathbf{U}}^{\prime},
$$

where

$$
\begin{aligned}
& \mathbf{U}_{0}^{\prime}=\frac{\partial}{\partial \varphi^{\prime}}, \quad \tilde{\mathbf{U}}^{\prime}=b_{1}\left(\rho^{\prime}, \varphi^{\prime}\right) \frac{\partial}{\partial \rho^{\prime}}+b_{2}\left(\rho^{\prime}, \varphi^{\prime}\right) \frac{\partial}{\partial \varphi^{\prime}}, \\
& b_{1}\left(\rho^{\prime}, \varphi^{\prime}\right)=\left(\rho^{\prime}\right)^{3}\left(\frac{1}{8} \sin 4 \varphi^{\prime}-\frac{1}{4} \sin 2 \varphi^{\prime}\right),
\end{aligned}
$$




$$
b_{2}\left(\rho^{\prime}, \varphi^{\prime}\right)=\left(\rho^{\prime}\right)^{2}\left(\frac{3}{8}+\frac{1}{8} \cos 4 \varphi^{\prime}-\frac{1}{2} \cos 2 \varphi^{\prime}\right) .
$$

Let us apply the asymptotic decomposition algorithm to system (28) with averaging on group $S O(2)$, defined as

$$
\langle f(\rho, \varphi)\rangle \equiv \frac{1}{2 \pi} \int_{0}^{2 \pi} f(\rho, \varphi) d \varphi .
$$

We restrict ourselves by the first approximation and consider the operator equation

$$
\left[\mathbf{U}, \mathbf{S}_{1}\right]=\tilde{\mathbf{U}}-\operatorname{pr} \tilde{\mathbf{U}}
$$

where $\mathbf{S}_{1}=\gamma_{1}(\rho, \varphi) \partial / \partial \rho+\gamma_{2}(\rho, \varphi) \partial / \partial \varphi$. Compute the average value of coefficients (29). According to the general theory,

$$
\operatorname{pr} \tilde{\mathbf{U}}=\frac{3}{8} \rho^{2} \frac{\partial}{\partial \varphi} .
$$

Therefore, the centralized (averaged) system in the first approximation takes the form

$$
\dot{\rho}=0, \quad \dot{\varphi}=1+\varepsilon \frac{3}{8} \rho^{2}
$$

Operator equation (30) is replaced by the system of differential equations

$$
\frac{\partial \gamma_{j}}{\partial \varphi}=b_{j}(\rho, \varphi)+\left\langle b_{j}(\rho, \varphi)\right\rangle, j=1,2
$$

Written out, such systems are easily integrated into trigonometric functions.

\section{Examples: the Motion of a Point on a Sphere}

3.1. Algorithm of the asymptotic decomposition method in the space of homogeneous polynomials (group GL(3)). Consider the system of equations

$$
\begin{aligned}
& \dot{x}_{1}^{\prime}=x_{2}^{\prime}-\varepsilon\left(x_{1}^{\prime 2}-x_{3}^{\prime 2}\right), \\
& \dot{x}_{2}^{\prime}=x_{3}^{\prime}-x_{1}^{\prime}-\varepsilon 2\left(x_{1}^{\prime} x_{2}^{\prime}+x_{2}^{\prime} x_{3}^{\prime}\right), \\
& \dot{x}_{3}^{\prime}=-x_{2}^{\prime}+\varepsilon\left(x_{2}^{\prime}+\left(x_{1}^{\prime 2}-x_{3}^{\prime 2}\right)\right) .
\end{aligned}
$$

Let us write down the operator associated with system (31)

$$
\mathbf{U}_{0}^{\prime}=\mathbf{U}^{\prime}-\varepsilon \widetilde{\mathbf{U}}^{\prime}
$$

where

$$
\begin{aligned}
& \mathbf{U}^{\prime}=x_{2}^{\prime} \frac{\partial}{\partial x_{1}^{\prime}}+\left(x_{3}^{\prime}-x_{1}^{\prime}\right) \frac{\partial}{\partial x_{2}^{\prime}}-x_{2}^{\prime} \frac{\partial}{\partial x_{3}^{\prime}} \\
& \widetilde{\mathbf{U}}^{\prime}=\left(x_{1}^{\prime 2}-x_{3}^{\prime 2}\right) \frac{\partial}{\partial x_{1}^{\prime}}+2\left(x_{1}^{\prime} x_{2}^{\prime}+x_{2}^{\prime} x_{3}^{\prime}\right) \frac{\partial}{\partial x_{2}^{\prime}}+\left(x_{2}^{\prime}+\left(x_{1}^{\prime 2}-x_{3}^{\prime 2}\right)\right) \frac{\partial}{\partial x_{3}^{\prime}} .
\end{aligned}
$$


Making use of the notations introduced in Subsection 2.1, let us write down

$$
\mathbf{U}^{\prime}=\hat{\hat{x}}_{m_{1}}^{\prime} \mathcal{F} \partial^{\prime}, \quad \tilde{\mathbf{U}}^{\prime}=\hat{\hat{x}}_{m_{1}}^{\prime} \mathcal{Q}_{1} \partial^{\prime}+\hat{\hat{x}}_{m_{2}}^{\prime} \mathcal{Q}_{2} \partial^{\prime}
$$

We restrict ourselves to computation of the first approximation. The transformed operator has only two summands

$$
\mathbf{U}_{0}=\mathbf{U}+\varepsilon \mathbf{N}_{1} .
$$

In that case, to find operators $\mathbf{S}_{1}$ of the transformation and the operator $\mathbf{N}_{1}$ of the centralizer, one should consider the single operator equation

$$
\left[\mathbf{U}, \mathbf{S}_{1}\right]=\widetilde{\mathbf{U}}-\operatorname{pr} \widetilde{\mathbf{U}}
$$

We omit intermediate calculations and give a final form of the centralized system

$$
\begin{aligned}
& \dot{x}_{1}=x_{2}-\varepsilon\left(-\frac{1}{4} x_{2}+\left(x_{1}^{2}-x_{3}^{2}\right)\right), \\
& \dot{x}_{2}=x_{3}-x_{1}-\varepsilon\left(-\frac{1}{4} x_{3}+\frac{1}{4} x_{1}+2\left(x_{1} x_{2}+x_{2} x_{3}\right)\right), \\
& \dot{x}_{3}=-x_{2}-\varepsilon\left(\frac{1}{4} x_{2}-\left(x_{1}^{2}-x_{3}^{2}\right)\right) .
\end{aligned}
$$

Further investigations of system (32) consist in the selection of fast and slow variables. After the change of variables

$$
y_{1}=x_{1}+x_{3}, \quad y_{2}=x_{1}^{2}+x_{2}^{2}+x_{3}^{2}, \quad y_{3}=\frac{1}{\sqrt{2}} \operatorname{arccot} \frac{x_{3}-x_{1}}{\sqrt{2} x_{2}}
$$

it turns into

$$
\dot{y}_{1}=0, \quad \dot{y}_{2}=-\varepsilon 2\left(2 y_{2}-y_{1}^{2}\right) y_{1}, \quad \dot{y}_{3}=1+\frac{1}{4} \varepsilon .
$$

3.2. Asymptotical decomposition algorithm for a perturbed motion on $\mathrm{SO}(3)$. Again consider the problem of motion of a point on a sphere (31). The operator $\mathbf{U}^{\prime}$ can be represented as a sum of two operators $\mathbf{U}_{1}^{\prime}, \mathbf{U}_{2}^{\prime}$

$$
\mathbf{U}_{1}^{\prime}=x_{2}^{\prime} \frac{\partial}{\partial x_{1}^{\prime}}-x_{1}^{\prime} \frac{\partial}{\partial x_{2}^{\prime}}, \quad \mathbf{U}_{2}^{\prime}=x_{3}^{\prime} \frac{\partial}{\partial x_{2}^{\prime}}-x_{2}^{\prime} \frac{\partial}{\partial x_{3}^{\prime}} \text {. }
$$

Calculate the Poisson bracket of the operators $\mathbf{U}_{1}^{\prime}, \mathbf{U}_{2}^{\prime}$ and denote the result by $\mathbf{U}_{3}^{\prime}$

$$
\mathbf{U}_{3}^{\prime}={ }_{\text {def }}\left[\mathbf{U}_{1}^{\prime}, \mathbf{U}_{2}^{\prime}\right]=x_{1}^{\prime} \frac{\partial}{\partial x_{3}^{\prime}}-x_{3}^{\prime} \frac{\partial}{\partial x_{1}^{\prime}} .
$$

The operators $\mathbf{U}_{1}^{\prime}, \mathbf{U}_{2}^{\prime}, \mathbf{U}_{3}^{\prime}$ yield the finite-dimensional Lie algebra $\mathcal{B}_{3}$ as the relations

$$
\left[\mathbf{U}_{2}^{\prime}, \mathbf{U}_{3}^{\prime}\right]=\mathbf{U}_{1}^{\prime}, \quad\left[\mathbf{U}_{3}^{\prime}, \mathbf{U}_{1}^{\prime}\right]=\mathbf{U}_{2}^{\prime} .
$$

imply.This algebra is one of the group $S O(3)$ of three-dimensional space rotations. Thus, the solution of the zero approximation system obtained from the perturbed system (31) for $\varepsilon=0$, can be written as the Lie series

$$
x_{j}^{\prime}=e^{t\left(\mathbf{U}_{1}+\mathbf{U}_{2}\right)} x_{j}, \quad j=\overline{1,3} .
$$


Otherwise speaking, the solution (33) of the zero approximation system is an element of $S O(3)$. Use this fact while asymptotic decomposing system (31) in the representation space of $S O(3)$. The Hilbert space $T_{S O(3)}$ of the basic spherical functions can be considered. A function on $S O(3)$ in the linear space $T_{S O(3)}$ is represented by the convergent series

$$
f^{\prime}=\sum_{l=0}^{\infty} \sum_{m=-l}^{l} C_{l}^{m} Y_{l}^{m^{\prime}} \text {, where } C_{l}^{m}=\frac{1}{4 \pi} \int_{0}^{2 \pi} \int_{0}^{\pi} f^{\prime} \bar{Y}_{l}^{m^{\prime}} \sin \theta^{\prime} d \theta^{\prime} d \varphi^{\prime} .
$$

Here $Y_{l}^{m^{\prime}}$ denotes basic spherical functions (see [19]).

To use the representation space $T_{S O(3)}$, we should pass to the new variables $\rho^{\prime}, \varphi^{\prime}, \theta^{\prime}$ in the initial system (31).

$$
x_{1}^{\prime}=\rho^{\prime} \sin \theta^{\prime} \cos \varphi^{\prime}, x_{2}^{\prime}=\rho^{\prime} \sin \theta^{\prime} \sin \varphi^{\prime}, x_{3}^{\prime}=\rho^{\prime} \cos \theta^{\prime}, \rho^{\prime}=\sqrt{x_{1}^{\prime 2}+x_{2}^{\prime 2}+x_{3}^{\prime 2}} .
$$

In these variables, $\mathbf{U}_{1}^{\prime}, \mathbf{U}_{2}^{\prime}, \mathbf{U}_{3}^{\prime}$ take the form

$$
\begin{aligned}
& \mathbf{U}_{1}^{\prime}=-\frac{\partial}{\partial \varphi^{\prime}}, \mathbf{U}_{2}^{\prime}=\sin \varphi^{\prime} \frac{\partial}{\partial \theta^{\prime}}+\cot \theta^{\prime} \cos \varphi^{\prime} \frac{\partial}{\partial \varphi^{\prime}}, \\
& \mathbf{U}_{3}^{\prime}=-\cos \varphi^{\prime} \frac{\partial}{\partial \theta^{\prime}}+\cot \theta^{\prime} \cos \varphi^{\prime} \frac{\partial}{\partial \varphi^{\prime}} .
\end{aligned}
$$

Write the operators $\mathbf{L}_{1}^{\prime}, \mathbf{L}_{2}^{\prime}, \mathbf{L}_{3}^{\prime}$

$$
\begin{aligned}
& \mathbf{L}_{1}^{\prime}=\frac{-\sin \varphi^{\prime}}{\rho^{\prime} \sin \theta^{\prime}} \frac{\partial}{\partial \varphi^{\prime}}+\frac{1}{\rho^{\prime}} \cos \theta^{\prime} \cos \varphi^{\prime} \frac{\partial}{\partial \theta^{\prime}}+\sin \theta^{\prime} \cos \varphi^{\prime} \frac{\partial}{\partial \rho^{\prime}}, \\
& \mathbf{L}_{2}^{\prime}=\frac{\cos \varphi^{\prime}}{\rho^{\prime} \sin \theta^{\prime}} \frac{\partial}{\partial \varphi^{\prime}}+\frac{1}{\rho^{\prime}} \cos \theta^{\prime} \sin \varphi^{\prime} \frac{\partial}{\partial \theta^{\prime}}+\sin \theta^{\prime} \sin \varphi^{\prime} \frac{\partial}{\partial \rho^{\prime}} \\
& \mathbf{L}_{3}^{\prime}=\frac{-\sin \theta^{\prime}}{\rho^{\prime}} \frac{\partial}{\partial \theta^{\prime}}+\cos \theta^{\prime} \frac{\partial}{\partial \rho^{\prime}},
\end{aligned}
$$

corresponding to the operators of partial differentiation $\partial / \partial x_{1}, \partial / \partial x_{2}, \partial / \partial x_{3}$ in new variables.

The operator $\mathbf{U}^{\prime}$ associated with the zero approximation system can be written in new variables as

$$
\mathbf{U}_{f}^{\prime}=f^{\left(m_{1}\right)^{\prime}} \mathcal{F} L^{\prime}
$$

where

$$
\begin{aligned}
& f^{\left(m_{1}\right)^{\prime}}=\left\|\rho^{\prime} \sin \theta^{\prime} \cos \varphi^{\prime}, \rho^{\prime} \sin \theta^{\prime} \sin \varphi^{\prime}, \rho^{\prime} \cos \theta^{\prime}\right\|, \\
& L^{\prime}=\operatorname{col}\left\|\mathbf{L}_{1}^{\prime}, \mathbf{L}_{2}^{\prime}, \mathbf{L}_{3}^{\prime}\right\| .
\end{aligned}
$$

The perturbation operator $\tilde{\mathbf{U}}_{f}^{\prime}$ in new variables can be represented as a sum of two operators

$$
\tilde{\mathbf{U}}_{f}^{\prime}=\tilde{\mathbf{U}}_{f 1}^{\prime}+\tilde{\mathbf{U}}_{f 2}^{\prime}
$$


where

$$
\tilde{\mathbf{U}}_{f 1}^{\prime}=f^{(1)^{\prime}} \mathcal{Q}_{m_{1}, 1} L^{\prime}, \quad \tilde{\mathbf{U}}_{f 2}^{\prime}=f^{(2)^{\prime}} \mathcal{Q}_{m_{2}, 1} L^{\prime} .
$$

By the operator

$$
\mathbf{U}_{f 0}^{\prime}=\mathbf{U}_{f}^{\prime}-\varepsilon \tilde{\mathbf{U}}_{f}^{\prime},
$$

we can easily restore the perturbed system (31) in new variables

$$
\begin{aligned}
& \dot{\varphi}^{\prime}=-1+\cot \theta^{\prime} \cos \varphi^{\prime}+\varepsilon \tilde{U}_{\varphi}^{\prime}, \\
& \dot{\theta}=\sin \varphi^{\prime}+\varepsilon \tilde{U}_{\theta}^{\prime}, \\
& \dot{\rho}=\varepsilon \tilde{U}_{\rho}^{\prime},
\end{aligned}
$$

where

$$
\tilde{U}_{\varphi}^{\prime}=\tilde{\mathbf{U}}_{f}^{\prime} \varphi, \tilde{U}_{\theta}^{\prime}=\tilde{\mathbf{U}}_{f}^{\prime} \theta, \tilde{U}_{\rho}^{\prime}=\tilde{\mathbf{U}}_{f}^{\prime} \rho
$$

are known functions.

The techniques of application of the asymptotic decomposition algorithm in the first approximation to system (34) in general is like to described above. Hence, we may cite here the final form of the centalised system

We restore $\mathbf{N}_{1}$ from algebra of centralizer using the matrices pr $\mathcal{Q}_{m_{1}, 1}$, pr $\mathcal{Q}_{m_{2}, 1}$

$$
\mathbf{N}_{1}=\mathbf{N}_{11}+\mathbf{N}_{21},
$$

where

$$
\mathbf{N}_{11}=f^{(1)} \operatorname{pr} \mathcal{Q}_{m_{1}, 1} L, \mathbf{N}_{21}=f^{(2)} \operatorname{pr} \mathcal{Q}_{m_{2}, 1} L .
$$

Using operator $N_{1}$, we restore the centralized system of the first approximation

$$
\begin{aligned}
& \dot{\varphi}_{1}=-1+\cot \theta \cos \varphi+\varepsilon N_{1 \varphi}, \\
& \dot{\theta}=\sin \varphi+\varepsilon N_{1 \theta}, \\
& \dot{\rho}=\varepsilon N_{1 \rho},
\end{aligned}
$$

where

$$
N_{1 \varphi}=\mathbf{N}_{1} \varphi, N_{1 \theta}=\mathbf{N}_{1} \theta, N_{1 \rho}=\mathbf{N}_{1} \rho .
$$

3.3. Group averaging for a perturbed motion on $\mathrm{SO}(3)$. Let us consider system (34). Operator $U^{\prime}$ associated with the system of zero approximation of system (34)

$$
U^{\prime}=\left(-1+\cot \theta^{\prime} \cos \varphi^{\prime}\right) \frac{\partial}{\partial \varphi^{\prime}}+\sin \varphi^{\prime} \frac{\partial}{\partial \theta^{\prime}}
$$

does not contain the variable $\rho^{\prime}$. 
We begin with some definitions which are fundamental for an algorithm of averaging on a noncommutative group. We assume that operators $S_{j}$ in series (14) have the form

$$
\mathbf{S}_{j}=\gamma_{j 3}(\varphi, \theta, \rho) \frac{\partial}{\partial \rho}, \quad \rho=1,2 \cdots
$$

The motivation of such a form of operators $\mathbf{S}_{j}$ will become clear some later. Operators $\mathbf{F}_{\nu}$ in the right part of equations (15) also can be written as a sum

$$
\mathbf{F}_{\nu}=\mathbf{F}_{\nu \rho}+\overline{\mathbf{F}}_{\nu}
$$

where

$$
\begin{aligned}
& \mathbf{F}_{\nu \rho}=f_{\nu \rho}(\varphi, \theta, \rho) \frac{\partial}{\partial \rho} \\
& \overline{\mathbf{F}}_{\nu}=f_{\nu \varphi}(\varphi, \theta, \rho) \frac{\partial}{\partial \varphi}+f_{\nu \theta}(\varphi, \theta, \rho) \frac{\partial}{\partial \theta} .
\end{aligned}
$$

The coefficients of $\mathbf{S}_{j}$ should be determined by the recurrent sequence of operator equations

$$
\left[\mathbf{U}, \mathbf{S}_{\nu}\right]=\mathbf{F}_{\nu \rho}-\mathbf{N}_{\nu \rho}
$$

Coefficient $f_{\nu \rho}(\varphi, \theta, \rho)$ of operator $\mathbf{F}_{\nu \rho}$ is a function on the group $S O(3)$ and hence may be written as a series

$$
f_{\nu \rho}=b_{\nu \rho 0}+f^{m 1} b_{\nu \rho 1}+f^{m 2} b_{\nu \rho 2}+\cdots,
$$

where $f^{m 1}, f^{m 2}, \cdots$ are vector-rows forms of bases of invariant subspaces and $b_{\nu \rho 0}, b_{\nu \rho 1}$, $b_{\nu \rho 2}, \cdots$ are vector-columns of coefficients.

Similarly, the coefficient $\gamma_{\nu 3}(\varphi, \theta, \rho)$ of the operator $\mathbf{S}_{\nu}$ can be written as

$$
\gamma_{\nu 3}=a_{\nu \rho 0}+f^{m 1} a_{\nu \rho 1}+f^{m 2} a_{\nu \rho 2}+\cdots,
$$

where $a_{\nu \rho 0}, a_{\nu \rho 1}, a_{\nu \rho 2}, \cdots$ are vector-columns of unknown coefficients.

Operator equations (35) produce the sequence of algebraic equations

$$
\begin{aligned}
& F_{1} a_{\nu \rho 1}=b_{\nu \rho 1} \\
& F_{2} a_{\nu \rho 1}=b_{\nu \rho 2}
\end{aligned}
$$

We suppose that equations (36) are solvable and

$$
\mathbf{N}_{\nu \rho}=b_{\nu \rho 0}(\rho) \frac{\partial}{\partial \rho},
$$

where

$$
b_{\nu \rho 0}(\rho)=\frac{1}{4 \pi} \int_{0}^{2 \pi} \int_{0}^{\pi} f_{\nu \rho}(\varphi, \theta, \rho) \bar{Y}_{l}^{m} \sin \theta d \theta d \varphi
$$

is an average value of the function $f_{\nu \rho}(\varphi, \theta, \rho)$ on the group $\mathrm{SO}(3)$. 
The operator associated with system (34) will have the form after all necessary transformations

$$
\mathbf{U}+\varepsilon\left(\mathbf{N}_{1 \rho}+\overline{\mathbf{F}}_{1}\right)+\varepsilon^{2}\left(\mathbf{N}_{2 \rho}+\overline{\mathbf{F}}_{2}\right)+\cdots .
$$

The following system corresponds to this operator

$$
\begin{aligned}
& \dot{\rho}=\varepsilon b_{1 \rho 0}(\rho)+(\varepsilon)^{2} b_{2 \rho 0}(\rho)+\cdots, \\
& \dot{\varphi}=-1+\cot \theta \cos \varphi+\varepsilon f_{1 \varphi}(\varphi, \theta, \rho)+(\varepsilon)^{2} f_{2 \varphi}(\varphi, \theta, \rho)+\cdots, \\
& \dot{\theta}=\sin \varphi+\varepsilon f_{1 \theta}(\varphi, \theta, \rho)+(\varepsilon)^{2} f_{2 \theta}(\varphi, \theta, \rho)+\cdots .
\end{aligned}
$$

The first equation of this system for a slow variable describes the parameter $\rho$ of the manifold. The next two equations describe angle variables of motion of a point on the manifold.

The algorithm of averaging on group given above is based on the further generalization of normalization techniques. Here we have partial commutativity of operators of the centralized system. Namely,

$$
\left[\mathbf{U}, \mathbf{N}_{\nu \rho}\right] \equiv 0
$$

but

$$
\left[\mathbf{U}, \overline{\mathbf{F}}_{\nu}\right] \not \equiv 0
$$

\section{References}

[1] Molchanov A.M., Separation of motions and asymptotic methods in the theory of linear oscillations (in Russian), DAN SSSR, 1961, V.5, 1030-1033.

[2] Brjuno A.D., Local Method of Nonlinear Analysis of Differential Eqs. (in Russian), Nauka, Moscow, 1979.

[3] Chow S.N., Mallet-Paret J., Integral averaging and bifurcation, J. Diff. Eqns., 1977, V.37, 351-373.

[4] Mitropolsky Yu.A., Samoilenko A.M., To the problem on asymptotic decompositions of nonlinear mechanics (in Russian), Ukr. Mat. Zhurn. 1976, V.31, N 1, 42-53.

[5] Sanders J.A., Verhulst F., Averaging Methods in Nonlinear Dynamical Systems, Springer-Verlag, New York, 1985.

[6] Hori G., Theory of general perturbations with unspecified canonical variables, J. Japan. Astron. Soc., 1966, V.18, N 4, 287-296.

[7] Hori G., Lie transformations in nonhamiltonian systems, Lecture notes, Summer Institute in Orbital Mechanics, Univ. Texas, Austin, 1970.

[8] Kamel A., A perturbations methods in the theory of nonlinear oscillations, Celest. Mech., 1970, V.3, N 1, 90-106.

[9] Kirchgraber U., Stiefel E., Methoden der Analytischen Storungsrechnung und Anwedungen (in German), Teubner, Anwedungen, Stuttgart, 1978. 
[10] Kirchgraber U., On the Lie-series approach to the method of averaging, In: IX Mezhdunar. Konf. po Nelinein. Kolebanijam, Naukova Dumka, Kiev, 1984, V.1, 173-178.

[11] Bogaevsky V.N., Povzner A.Ya., Algebraic Methods in Nonlinear Perturbation Theory (in Russian), Nauka, Moscow, 1987.

[12] Zhuravlev V.F., Klimov D.N., Applied Methods in the Oscillations Theory (in Russian), Nauka, Moscow, 1988.

[13] Mitropolsky Yu.A., Lopatin A.K., Group theory approach in asymptotic methods of nonlinear mechanics (in Russian), Naukova Dumka, Kiev, 1988.

[14] Bogolyubov's averaging and procedures of normalization in nonlinear mechanics I,II,III, Ukr. Mat. Zhurn., 1994, V.42, N 9, 1171-1188; N 11, 1509-1526; N 12, 1627-1646.

[15] Mitropolsky Yu.A., Lopatin A.K., Nonlinear Mechanics, Groups and Symmetry, Kluwer Academic Publisher, Dordrecht, Boston, London, 1995.

[16] Lopatin A.K., Averaging, normal forms and symmetry in nonlinear mechanics, Preprint, Inst. Mat. Nat. Acad. Ukrainy, Kiev, 1994.

[17] Averaging, normal forms and symmetry in nonlinear mechanics, Preprint, Inst. Mat. NAN Ukrainy, Kiev, 1994.

[18] Bogolyubov N.N., Mitropolsky Yu.A., Asymptotic Methods in the Theory of Nonlinear Oscillations, Nauka, Moscow, 1974; English transl., Gordon and Breach, New York, 1961.

[19] Vilenkin N.Ja., Special Functions and the Theory of Group Representations (in Russian), Second edition, Nauka, Moscow, 1992; English transl., Transl. Math. Monogr. vol. 23, American Mathematical Society, Providence, R.I., 1968.

[20] Barut A., Roczka R., Theory of Group Representations and its Applications, PWN, Warsaw, 1977; Russian transl., Mir, Moscow, 1980. 